\title{
Estimating cohort health expectancies from cross-sectional surveys of disability
}

\author{
B. A. Davis ${ }^{1}$, C. R. Heathcote ${ }^{2,3, *, \dagger}$, and T. J. O’Neill ${ }^{1}$ \\ ${ }^{1}$ Department of Statistics and Econometrics, Australian National University, Canberra ACT 0200, Australia \\ ${ }^{2}$ Centre for Mathematics and its Applications, Australian National University, Canberra ACT 0200, Australia \\ ${ }^{3}$ Department of Epidemiology and Public Health, University of Leicester, Leicester LE1 6TP, U.K.
}

\begin{abstract}
SUMMARY
A life history can be regarded as a random process that evolves with age through various states of health before terminating with absorption into the state of death. Health expectancies are the occupation times of the non-absorbing states and their estimation is of interest. A continuing major problem has been the lack of satisfactory longitudinal data on which to base estimates and as a result standard inferential techniques may not be relevant. Supposing only cross-sectional data available, we propose a method that is generally applicable and first estimates a logistic parametrization of the probabilities of the various states. A large sample approximation is obtained for the distribution of age specific $\log$ (odds). Parameters are estimated by weighted least squares, and this in turn leads to estimates of cohort health expectancies. A result of Liang and Zeger is used to find standard errors. The method is illustrated by application to Australian data from the health surveys of 1981, 1988 and 1993. Copyright (C) 2001 John Wiley \& Sons, Ltd.
\end{abstract}

\section{INTRODUCTION}

Health expectancies are components of life expectancy in the sense that they are the future durations that an individual expects to spend in different states of health. As such they are of interest to students of public health and can be used as summary measures of the well-being of a population. Useful references are Katz et al. [1], Bebbington [2], Robine and Ritchie [3] and Mathers et al [4].

Mathers [5] gives a summary review of developments to 1996 and, together with an earlier work [6], applies what is the most popular method of calculation, due to Sullivan [7], to the Australian disability surveys discussed in Section 3. Sullivan's method contains both period and cohort considerations but predominantly the former. The cohort element arises typically from surveys designed to estimate prevalences of health states whereas the period component is due to the use of standard life tables. We shall be concerned with data originating from official statistics in which counts of numbers of individuals in different health states are available from

\footnotetext{
${ }^{*}$ Correspondence to: C. R. Heathcote, Centre for Mathematics and its Applications, Australian National University, Canberra ACT 0200, Australia

† E-mail: heacstat@maths.anu.edu.au
} 
cross-sectional investigations at a few points in time. Our aim is to present a method which is an alternative to that of Sullivan and that uses the cross-sectional information to attempt a reconstruction of relevant parts of the underlying longitudinal process. The proposed method is not specific to health data and is of general applicability. For other discussions of variants of the problem see, for example, Deaton [8], and MacRae [9].

Estimation of health expectancies generally requires data on the numbers of transitions between states, such data being available from longitudinal studies. However, the situation in which only cross-sectional data is available is not uncommon in the medical and social sciences since it is frequently the case that cross-sections of a population are easier and cheaper to sample at a sequence of time points than the alternative of implementing a more informative longitudinal study. A considerable statistical literature exists on the analysis of cross-sectional data. Some examples of recent interest are Gargiullo et al. [10]. Martuzzi and Elliot [11], Thompson et al. [12], Skov et al. [13] and Zocchetti et al. [14]. The use of repeated cross-sectional studies does not seem to have been widely considered.

The case considered here is a three-state system and we will say that an individual is in state 1 if they are free of disability (however defined), in state 2 if they are disabled and in state 3 if dead. Given that a person is alive at age $x$, the problem is to estimate the health expectancies of states 1 and 2, that is, the time expected to be spent in the states of disability-free and disabled between age $x$ and death. Health expectancies are defined more precisely in the next section. Sections 3 and 4 concern the estimation of health expectancies at selected ages from data obtained by the Australian disability surveys of 1981, 1988 and 1993, with the main numerical results presented in Table III. The surveys were large cross-sectional population studies and they are used here to construct estimates of cohort health expectancies. Section 5 and the Appendix treat methodological issues.

\section{HEALTH EXPECTANCIES}

Let $p(x+y)$ denote the conditional probability that an individual is alive at age $x+y$ given that they were alive at age $x$. Then life expectancy at age $x$ is

$$
e(x)=\sum_{y=0}^{\infty} p(x+y)
$$

Supposing that the status of being alive is partitioned exhaustively into $k$ 'health' states, numbered $1, \ldots, k$, let $p_{i}(x+y)$ be the probability of being in state in $i$ at age $x+y$ conditional on being alive at $x$. Then

$$
e_{i}(x)=\sum_{y=0}^{\infty} p_{i}(x+y)
$$

is the (marginal) health expectancy of state $i$ at age $x$. It is a marginal quantity with respect to the particular health state at age $x$. We refer to the $e_{i}(x)$ simply as health expectancies at $x$ and the estimation from cross-sectional data of $e_{1}(x)$ and $e_{2}(x)$ in the case $k=2$ is the problem considered in this paper.

Truncating the summation in (1) at age $x_{1}$ will underestimate a health expectancy but not by much if $x_{1}$ is high. Owing to reservations about extrapolation and the quality of some 
of the data at old ages, the numerical results we give in Section 4 take $x_{1}=95$. Thus we consider health expectancies at ages $x$ to age 95 , but continue to refer to them simply as health expectancies.

There are period and cohort health (and life) expectancies just as there are period and cohort life tables. With superscript $\mathrm{p}$ denoting 'period' suppose, for a given year, we have a standard one year age increment period life table with

$$
\begin{aligned}
& \ell(x)^{(\mathrm{p})}=\text { number of lives at age } x \\
& L(x)^{(\mathrm{p})}=\text { total number of years lived in }(x, x+1)
\end{aligned}
$$

Then at the year in question, period life expectancy at age $x$ is estimated by

$$
e(x)^{(\mathrm{p})}=\sum_{y=0}^{\infty} L(x+y)^{(\mathrm{p})} / \ell(x)^{(\mathrm{p})}
$$

and similar estimates of period health expectancies are obtained on replacing $L(x+y)^{(\mathrm{p})}$ by $L_{i}(x+y)$, the number of years spent in state $i$ during $(x+y, x+y+1)$. It is the fact that this latter quantity is seldom observed directly that gives rise to many of the difficulties in estimating health expectancies. The commonly used Sullivan estimate of health expectancies substitutes $\pi_{i}(x+y) L(x+y)^{(\mathrm{p})}$ in place of $L_{i}(x+y)$, where $\pi_{i}(x+y)$ is an estimate of the prevalence of state $i$ at age $x+y$. This estimate of prevalence is typically obtained from a survey and what is known as the Sullivan estimate of the health expectancy of state $i$ at age $x$ is

$$
e_{i}(x)^{(\mathrm{s})}=\sum_{y=0}^{\infty} \pi_{i}(x+y) L(x+y)^{(\mathrm{p})} / \ell(x)^{(\mathrm{p})}
$$

For an extensive discussion see Mathers [6], Bebbington [2] or Mathers et al. [4].

The $e_{i}(x)^{(\mathrm{s})}$ are essentially period constructs, that is they are specific to a particular year, since period life tables were used in their calculation. Corresponding cohort (by which we mean birth cohort) expectancies could be estimated in the same way if cohort life tables were available, but this is seldom the case for populations of interest. However, estimates of cohort health expectancies are of importance to persons now living and to planners of future health services. By definition the Sullivan method as described cannot supply these estimates, except in so far that a period measure is a surrogate for the analogous cohort quantity. We argue that a totally different approach may be helpful.

From the point of view of this paper, a life history, which is a cohort concept, is a process consisting of sequential durations of time, or age, spent in different states of functional disability before terminating in the absorbing state of death. A health expectancy $e_{i}(x)$ is then the expected future occupation time of state $i$ at age $x$. Given perhaps only fragmentary information about the temporal evolution of a cohort of individuals, the general problem is to uncover the mechanism generating the process. Lack of data usually prevents the use of multistate life table methodology as in Land et al. [15] but the assumptions underlying these methods must generally be accepted. Of importance is the assumption of homogeneity in the sense that individuals in the same cohort independently obey the same probabilistic laws and that cohorts are stochastically independent. 
The assumption of homogeneity underlies the classical distribution theory of life tables; see for example Part 2 of Chiang [16]. Just as this leads to the binomial distribution for the number of survivors of a given age in the ordinary cohort life table, so it implies a multinomial distribution of frequencies in the multistate case. As developed in the Appendix, the multinomial distribution and its approximation by a multivariate normal provides the basis for the regression type arguments used in this paper (a general theoretical treatment will be given elsewhere). We return to the issue of homogeneity later when discussing standard errors but retain the assumption for the purposes of deriving point estimates of health expectancies.

From (1), estimating the $p_{i}(x+y)$ is a natural first step in the estimation of health expectancies. Writing

$$
\begin{aligned}
& \ell_{i}(x)=\text { number of lives in state } i \text { at age } x \\
& \ell(x)=\sum_{i} \ell_{i}(x)=\text { total number of lives at } x
\end{aligned}
$$

we have

$$
p_{i}(x+y)=\ell_{i}(x+y) / \ell(x)
$$

Thus properties of estimators of the frequencies on the right hand side determine what can be inferred about the probabilities and hence the health expectancies.

The procedure used in this paper estimates the probabilities through the odds of a state with respect to a reference state. To be precise, if state 1 (disability-free) is the reference state, let, for $i=2,3$

$$
\xi_{i}(y)=\log \left\{p_{i}(y) / p_{1}(y)\right\}=\log \left\{\ell_{i}(y) / \ell_{1}(y)\right\}
$$

be the logarithm of the odds of state $i$ relative to state 1 at age $y$. Since the probabilities sum to unity, (4) forces the logistic form

$$
\begin{aligned}
& p_{1}(y)=\left\{1+\sum_{i=2}^{3} \mathrm{e}^{\xi_{i}(y)}\right\}^{-1} \\
& p_{i}(y)=p_{1}(y) \mathrm{e}^{\xi_{i}(y)}, \quad i=2,3
\end{aligned}
$$

Parameterized $p_{i}(y)$, and hence the health expectancies, will be estimated in terms of regression models of the $\xi_{i}(y)$ using the theory of Section 5 and applied to the data described in the next section.

\section{THE AUSTRALIAN DISABILITY SURVEYS}

The data now discussed originate from the ageing and disability surveys carried out by the Australian Bureau of Statistics (ABS) in 1981, 1988 and 1993. We refer to Mathers [5, 6] for a detailed description and discussion of the surveys. Here, it is sufficient to note the following points. They were population surveys with sample sizes of about 75000 each for 
Table I. Australian females, 1911-1915 cohorts, aged 65 years in 1976-1980. Numbers of disability-free, disabled and dead, respectively, at age $y$ in the survey years 1981, 1988, 1993.

\begin{tabular}{|c|c|c|c|c|c|c|}
\hline \multirow[t]{2}{*}{ Cohort } & \multicolumn{2}{|c|}{1981} & \multicolumn{2}{|c|}{1988} & \multicolumn{2}{|c|}{1993} \\
\hline & $y$ & $\ell_{i}(y)$ & $y$ & $\ell_{i}(y)$ & $y$ & $\ell_{i}(y)$ \\
\hline $1915 \ell(65)=59940$ in 1980 & 66 & $\begin{array}{r}42180 \\
17573 \\
187\end{array}$ & 73 & $\begin{array}{r}27586 \\
25119 \\
7235\end{array}$ & 78 & $\begin{array}{l}19626 \\
25037 \\
15277\end{array}$ \\
\hline $1914 \ell(65)=60152$ in 1979 & 67 & $\begin{array}{r}39796 \\
18386 \\
1970\end{array}$ & 74 & $\begin{array}{r}26212 \\
25663 \\
8277\end{array}$ & 79 & $\begin{array}{l}18035 \\
24813 \\
17304\end{array}$ \\
\hline $1913 \ell(65)=57738$ in 1978 & 68 & $\begin{array}{r}37379 \\
17630 \\
2729\end{array}$ & 75 & $\begin{array}{l}23334 \\
24132 \\
10272\end{array}$ & 80 & $\begin{array}{l}15394 \\
22887 \\
19457\end{array}$ \\
\hline $1912 \ell(65)=56401$ in 1977 & 69 & $\begin{array}{r}36535 \\
16484 \\
3382\end{array}$ & 76 & $\begin{array}{l}20671 \\
23423 \\
12307\end{array}$ & 81 & $\begin{array}{l}13370 \\
21533 \\
21498\end{array}$ \\
\hline $1911 \ell(65)=56535$ in 1976 & 70 & $\begin{array}{r}35332 \\
17775 \\
3428\end{array}$ & 77 & $\begin{array}{l}19283 \\
22710 \\
14542\end{array}$ & 82 & $\begin{array}{l}11670 \\
20413 \\
24452\end{array}$ \\
\hline
\end{tabular}

the first two and about 45000 for the third. Interviews were conducted by trained personnel but the identification of disability and its severity relied on self-assessment. There was no longitudinal component and the surveys can be regarded as three independent samplings of functional health in the country. The definition of disability was based on the WHO International Classification of Impairments Disabilities and Handicaps and meant that a disabled person suffered from at least one of a list of specified conditions, such as loss of sight or hearing, for a period of at least six months. Also slight differences in the questionnaires were taken into account so that relevant results from the three surveys are compatible.

Sullivan's formula (2) was used by Mathers [5,6] to calculate cross-sectional prevalences and health expectancies by single years of age for 1981 and 1988 and in less detail for 1993 . Some further data on the 1993 survey held by the Australian Institute of Health and Welfare were kindly made available and the frequencies of health states, such as in Table I and II, that provide the data to which our methods are applied are based on Mather's prevalences combined with the appropriate ABS population counts. It is believed that some of the original data have been lost so there seems no alternative way of obtaining the frequencies of the health states. Thus we are in a situation not uncommon to users of official statistics in that the data available to us has been subject to some form of graduation. This artificial component should be borne in mind since it is likely to lead to the underestimation of standard errors.

Whilst the method developed in Section 5 is of wide applicability, the cohort health expectancies tabulated in the next section are for persons who were aged between 63 and 77 years in 1980. The year 1980 was selected as de facto (time, age) origin so that data from all three surveys, commencing in 1981, could be used in the estimation procedure. Thus the health status of a person alive and of age $x$ in 1980 can be sampled at age $x+1$ in 1981, at 
Table II. Australian males, 1911-1915 cohorts, aged 65 years in 1976-1980. Numbers of disability-free, disabled and dead, respectively, at age $y$ in the survey years 1981, 1988, 1993.

\begin{tabular}{|c|c|c|c|c|c|c|}
\hline \multirow[t]{2}{*}{ Cohort } & \multicolumn{2}{|c|}{1981} & \multicolumn{2}{|c|}{1988} & \multicolumn{2}{|c|}{1993} \\
\hline & $y$ & $\ell_{i}(y)$ & $y$ & $\ell_{i}(y)$ & $y$ & $\ell_{i}(y)$ \\
\hline $1915 \ell(65)=54014$ in 1980 & 66 & $\begin{array}{r}33733 \\
19323 \\
958\end{array}$ & 73 & $\begin{array}{l}20557 \\
20780 \\
12677\end{array}$ & 78 & $\begin{array}{l}10931 \\
19945 \\
23138\end{array}$ \\
\hline $1914 \ell(65)=54272$ in 1979 & 67 & $\begin{array}{r}33578 \\
17914 \\
2780\end{array}$ & 74 & $\begin{array}{l}18819 \\
20274 \\
15179\end{array}$ & 79 & $\begin{array}{r}9496 \\
18578 \\
26198\end{array}$ \\
\hline $1913 \ell(65)=51532$ in 1978 & 68 & $\begin{array}{r}31200 \\
16016 \\
4316\end{array}$ & 75 & $\begin{array}{l}17007 \\
18227 \\
16298\end{array}$ & 80 & $\begin{array}{r}7900 \\
16600 \\
27032\end{array}$ \\
\hline $1912 \ell(65)=51023$ in 1977 & 69 & $\begin{array}{r}29214 \\
15717 \\
6092\end{array}$ & 76 & $\begin{array}{l}15099 \\
16351 \\
19573\end{array}$ & 81 & $\begin{array}{r}6517 \\
14733 \\
29773\end{array}$ \\
\hline $1911 \ell(65)=49732$ in 1976 & 70 & $\begin{array}{r}27008 \\
15021 \\
7703\end{array}$ & 77 & $\begin{array}{l}13554 \\
15271 \\
20907\end{array}$ & 82 & $\begin{array}{r}5358 \\
13061 \\
31313\end{array}$ \\
\hline
\end{tabular}

age $x+8$ in 1988 and at age $x+13$ in 1993, giving only three data vectors per initial age $x$ from which to estimate the probabilities $p_{i}(x+y)$.

To augment the number of points available for each initial age $x$ we make the working assumption that five-year age groups have probabilities with the same parameterization, giving a total of 15 data vectors for each $x$. For example, consider Table I concerning females aged 65 years in 1980. For this 1915 cohort, $\ell(65)=59940$ is the number of 65 -yearold females alive in 1980 (obtained from the ABS population count) and the data vectors $\ell(66)^{\prime}=(42180,17573,187), \ell(73)^{\prime}=(27586,25119,7235), \ell(78)^{\prime}=(19626,25037,15277)$ are the numbers of this 59940 who are respectively in the states disability-free, disabled, dead at the years of the three surveys. The counts of disabled and disability-free are obtained by multiplying the number alive by the appropriate prevalence of Appendix D of Mathers [6]. Other data vectors in the table concern females alive at age 65 years for the 1911-1914 cohorts and were found in the same way. Under the assumption that probabilities $p_{i}(x+y)$ for these five cohorts have the same parameters, we then have data for the 15 ages $66, \ldots, 70,73, \ldots, 82$ years from which to make inferences about the parameters of $p_{i}(65+y)$. For each initial age it is therefore necessary to construct two tables, one for each sex, similar to Tables I and II for age 65 years. To conserve space they are the only data tables displayed and the full data set is available on request.

\section{RESULTS}

The multinomial counts of Tables I and II for persons aged 65 years in 1980, and similar tables for other selected ages, were used to obtain the estimated health expectancies $e_{i}(x)$ of Table III. 
Table III. Estimated cohort health expectancies of the disability-free and disabled states and life expectancy for persons aged $x$ in 1980. Standard errors are in parentheses.

\begin{tabular}{cccccccc}
\hline$x$ & & & & & Males & \\
\cline { 2 - 3 } \cline { 7 - 7 } & $e_{1}(x)$ & $e_{2}(x)$ & $e(x)$ & & $e_{1}(x)$ & $e_{2}(x)$ & $e(x)$ \\
\hline 63 & 10.83 & 11.64 & 22.47 & & 8.46 & 10.59 & 19.05 \\
& $(0.263)$ & $(1.184)$ & $(1.443)$ & & $(0.100)$ & $(1.015)$ & $(1.055)$ \\
65 & 9.55 & 10.34 & 19.89 & & 7.59 & 8.82 & 16.41 \\
& $(0.072)$ & $(0.298)$ & $(0.319)$ & & $(0.053)$ & $(0.743)$ & $(0.729)$ \\
67 & 8.59 & 10.39 & 18.98 & & 6.79 & 7.77 & 14.56 \\
& $(0.065)$ & $(0.396)$ & $(0.444)$ & & $(0.050)$ & $(0.450)$ & $(0.459)$ \\
69 & 7.34 & 9.36 & 16.70 & & 5.82 & 6.89 & 12.71 \\
& $(0.086)$ & $(0.512)$ & $(0.596)$ & & $(0.088)$ & $(0.328)$ & $(0.405)$ \\
71 & 6.18 & 8.34 & 14.52 & & 4.91 & 6.30 & 11.21 \\
& $(0.051)$ & $(0.283)$ & $(0.319)$ & & $(0.025)$ & $(0.231)$ & $(0.239)$ \\
73 & 5.21 & 7.58 & 12.79 & & 4.23 & 5.69 & 9.92 \\
& $(0.032)$ & $(0.217)$ & $(0.244)$ & & $(0.031)$ & $(0.256)$ & $(0.228)$ \\
75 & 4.38 & 6.91 & 11.29 & & 3.71 & 5.06 & 8.77 \\
& $(0.028)$ & $(0.172)$ & $(0.173)$ & $(0.056)$ & $(0.222)$ & $(0.174)$ \\
77 & 3.62 & 6.32 & 9.94 & 3.23 & 4.54 & 7.77 \\
& $(0.028)$ & $(0.176)$ & $(0.170)$ & $(0.034)$ & $(0.188)$ & $(0.165)$ \\
\hline
\end{tabular}

The first step in the estimation procedure is the regression modelling of the $\log$ (odds) of (4) and Figures 1 and 2 for age 69 years are representative of the pattern obtained for most of the regressions. The first five observed values are from the 1981 survey, the next five from that of 1988, and the remaining five from 1993. A linear fit was found to be appropriate in the case of $\xi_{2}(y)$, that is $\log$ (odds) for disabled relative to disability-free, whereas a quadratic fit was found suitable for $\xi_{3}(y)$. Substitution of the fitted regressions into (5) gave estimates of the probabilities and hence, using (1), the health expectancies of Table III.

In Table III we see that $e_{2}(x)$ is greater than $e_{1}(x)$ for both sexes and all ages considered, indicating a substantial future burden of disability. The decrease in estimated disability-free health expectancy from 10.83 at age 63 to 3.62 at 77 for females, and the corresponding decline from 8.46 to 3.23 for males, suggests that there is an age linked sex difference in both the rate and magnitude of change in disability status. Perhaps a better summary measure of the latter is provided by the two columns headed 'Cohort' in Table IV. As age increases from 63 to 77 years, the percentage $100 \times e_{2}(x) / e(x)$ of future life spent in the disabled state is estimated to rise from about 52 per cent to 63.6 per cent for females. This is roughly double the increase for males for whom the estimated change is only five percentage points from 53.4 per cent to 58.4 per cent. In this sense the future burden of disability is greater for females than for males. The results are consistent with the expansion of morbidity hypothesis (see for example Olshansky et al. [17]) that at older ages disability increases as mortality decreases. However, it applies with greater force to females than males with the percentages of Table IV supplying numerical indicators.

An interesting side issue that we do not pursue here is the apparent 'retirement effect' in the male figures around the age 65 years, with disability seemingly diminishing for a period 

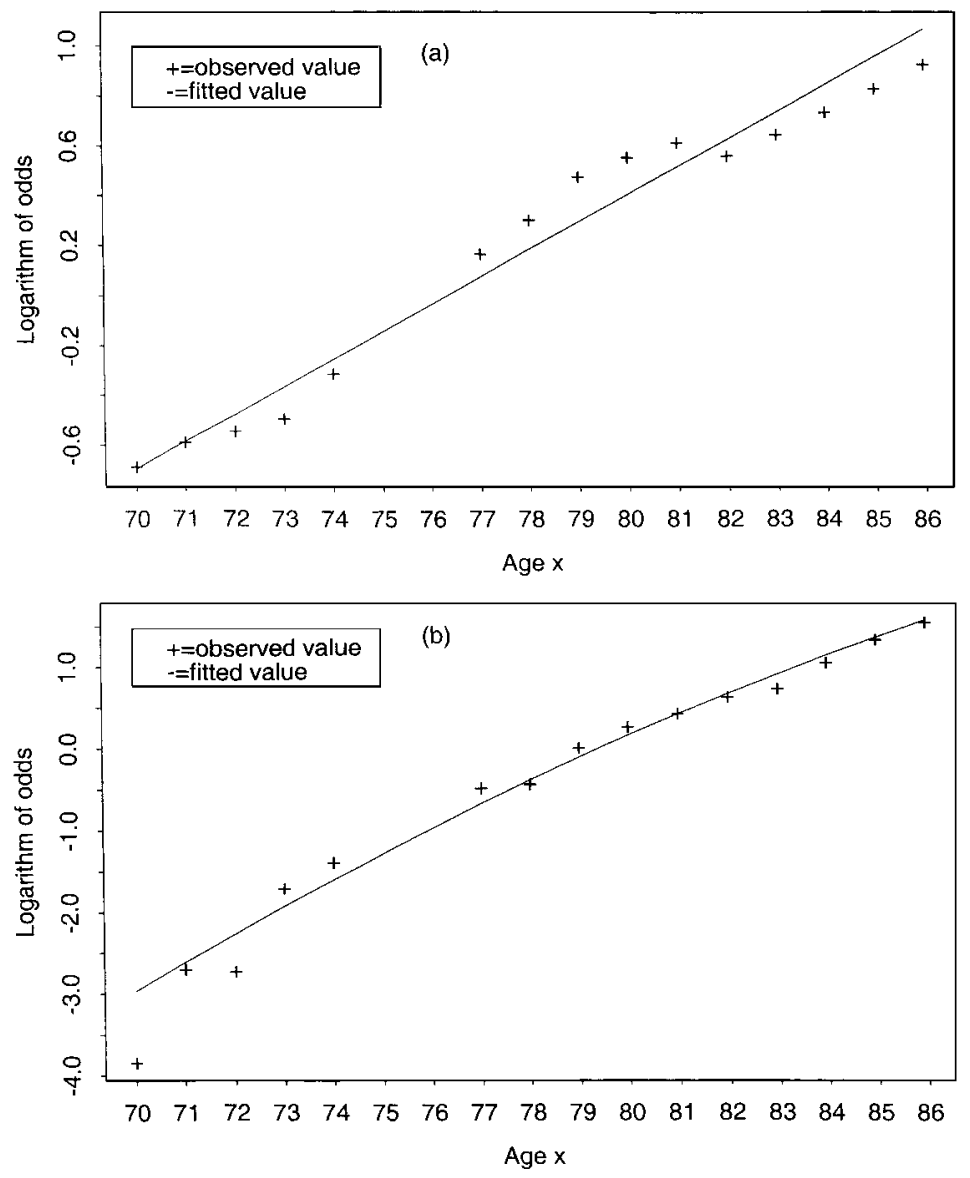

Figure 1. Australian females aged 69 years in year 1980: (a) fitted and observed log-odds of disability relative to disability-free; $(b)$ fitted and observed log-odds of death relative to disability-free.

after that age. In Australia, retirement at age 65 was, and largely still is, compulsory for males in the workforce and it seems that this event induces an improvement in health.

It should also be observed that in Table III all entries for females are higher than the same measure for males. Again the differences change with age. If superscript $\mathrm{F}$ stands for female and superscript $\mathrm{M}$ stands for male we see that $e_{1}(x)^{\mathrm{F}}-e_{1}(x)^{\mathrm{M}}$ decreases monotonically from 2.37 at age 63 years to 0.39 at 77 years, whereas $e_{2}(x)^{\mathrm{F}}-e_{2}(x)^{\mathrm{M}}$ remains bounded well away from zero, decreasing from a high of 2.62 at 67 to 1.78 at 77 years. Taking into account that the difference in life expectancy does not fall below two years, one concludes that the greater part of the extra years of life expected by females relative to males are ones of disability.

As cohort quantities, the entries of Table III were constructed using data from all three of the surveys in 1981, 1988 and 1993. Tables IV and V provide comparisons with analogous cross-sectional measures calculated by Sullivan's formula (2) for each survey separately. Not all details of the 1993 survey are available and it was not possible to include results for that year in Table IV. What is clear from the table is that the cohort measures are roughly 

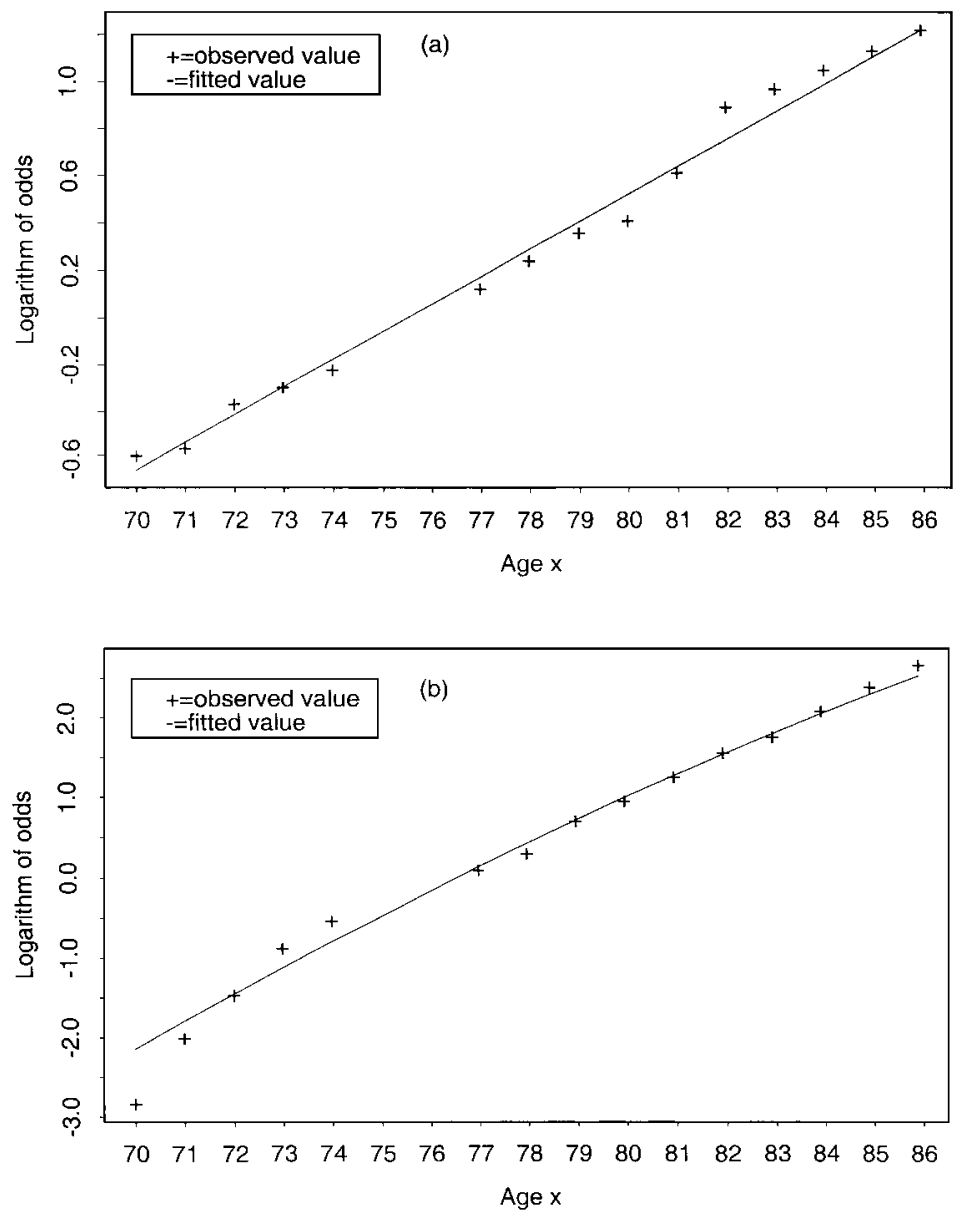

Figure 2. Australian males aged 69 years in year 1980: (a) fitted and observed log-odds of disability relative to disability-free; $(b)$ fitted and observed log-odds of death relative to disability-free.

an average of the analogous period quantities for 1981 and 1988, which is not unexpected, and that there is a striking difference in the results of the two surveys, which may give rise to surprise. It is beyond the scope of this paper to comment on the two surveys except to say that the matter has been taken up briefly elsewhere [6]. Changing community perceptions towards disability and welfare, rather than a real deterioration of health, are seen as possible explanations.

Another set of comparisons are given in Table V. Results for 65-year-olds were available from all three surveys with those for 1993 and 1988 generally forming a pair rather different in magnitude to the results of the 1981 survey. The numbers in the cohort row, from the row of Table III for persons aged 65 years in 1980, tend to be higher than the average of the cross-sectional entries, with cohort life expectancy significantly greater than the period life expectancies at the three survey years. On the other hand the proportion $e_{2}(x) / e(x)$ of future life expected to be spent in the disabled state retains the pattern of Table IV. Whatever the 
Table IV. Estimated percentages at age $x$ of future life spent in the disabled state. Entries under 'Cohort' are based on Table III and those under 1981, 1988 are from the Sullivan estimates (2) of Mathers [6].

\begin{tabular}{lccccccc}
\hline$x$ & \multicolumn{3}{c}{ Females } & & \multicolumn{3}{c}{ Males } \\
\cline { 2 - 3 } & Cohort & 1981 & 1988 & & Cohort & 1981 & 1988 \\
\hline 63 & 51.8 & 42.5 & 51.8 & 55.6 & 42.4 & 54.9 \\
65 & 52.0 & 44.3 & 54.1 & 53.7 & 43.3 & 55.0 \\
67 & 54.7 & 46.2 & 56.7 & 53.4 & 44.3 & 56.3 \\
69 & 56.1 & 48.2 & 59.3 & 54.2 & 46.1 & 57.8 \\
71 & 57.4 & 50.5 & 61.9 & 56.2 & 48.3 & 59.2 \\
73 & 59.3 & 52.9 & 64.6 & 57.4 & 50.5 & 60.8 \\
75 & 61.2 & 55.3 & 67.4 & 57.7 & 52.3 & 63.2 \\
77 & 63.6 & 57.6 & 70.6 & 58.4 & 54.1 & 66.2 \\
\hline
\end{tabular}

Table V. Cohort health expectancies for persons aged 65 years in 1980 and Sullivan estimates (2) for 65-year-olds from the surveys of 1981, 1988, 1993.

\begin{tabular}{|c|c|c|c|c|c|c|c|c|}
\hline & \multicolumn{4}{|c|}{ Females } & \multicolumn{4}{|c|}{ Males } \\
\hline & $e_{1}(65)$ & $e_{2}(65)$ & $e(65)$ & $e_{2} / e$ & $e_{1}(65)$ & $e_{2}(65)$ & $e(65)$ & $e_{2} / e$ \\
\hline Cohort & 9.55 & 10.34 & 19.89 & 0.520 & 7.59 & 8.82 & 16.41 & 0.537 \\
\hline 1981 & 10.07 & 8.00 & 18.07 & 0.443 & 7.87 & 6.00 & 13.87 & 0.433 \\
\hline 1988 & 8.58 & 10.13 & 18.71 & 0.541 & 6.65 & 8.13 & 14.77 & 0.550 \\
\hline 1993 & 9.09 & 10.39 & 19.48 & 0.533 & 6.51 & 9.22 & 15.73 & 0.586 \\
\hline
\end{tabular}

reasons for apparent inconsistencies between the surveys they provide the only data available and their use is therefore unavoidable. Furthermore the 'averaging' effect of working with cohorts can be argued to add to the attractiveness of the methodology we propose.

\section{METHODS}

In this section we adopt the notationally convenient convention that age $y$ is counted from some suitable origin. For example, if health expectancies at age $x_{0}$ are of interest then $y$ will count the number of years from $x_{0}$ with $y=0$ meaning age $x_{0}$. Sometimes, when there is no ambiguity, age will be omitted. Using this notation, consider a cohort of $\ell(0)$ with $\ell(0)$ large. For example, in Table I with $x_{0}=65$ the current $\ell(0)=59940$. More detailed initial conditions specify $\ell_{1}(0)$ and $\ell_{2}(0)$, the respective number in the two alive states at the commencement of observations, but these will generally be unknown. As introduced in Section 2, the symbol $\ell(y)$ denotes the number of lives at age $y, \ell_{i}(y), i=1,2$ the number in state $i$ at age $y$, and let $\ell_{3}(y)$ be the cumulative number of deaths up to the including age $y$. Then

$$
\ell_{1}(y)+\ell_{2}(y)+\ell_{3}(y)=\ell(0)
$$

For $y>0$ the frequencies $\ell_{i}(y)$ and $\ell(y)$ are unknown population quantities that can be estimated from observed counts. Thus the denominator of

$$
p_{i}(y)=\ell_{i}(y) / \ell(0)
$$

is known but the numerator must be estimated. 
The notation $\tilde{\ell}_{i}(y)$ will be used to denote the random variable whose expectation is $\ell_{i}(y)$

$$
E \tilde{\ell}_{i}(y)=\ell_{i}(y)
$$

and, for the probability of state $i$ at age $y$

$$
\tilde{p}_{i}(y)=\tilde{\ell}_{i}(y) / \ell(0)
$$

Superimposing a tilda on a letter indicates that it is a random variable with the same letter without a tilda being its expectation, or more generally its limit in probability.

The random variables estimating the $\log$ (odds) of (4) are

$$
\tilde{\xi}_{i}(y)=\log \left\{\tilde{p}_{i} / \tilde{p}_{1}\right\}=\log \left\{\tilde{\ell}_{i} / \tilde{\ell}_{1}\right\}=\tilde{\xi}_{i}
$$

where age $y$ has been omitted on the right hand side. Under the assumption of homogeneity the distribution of $\tilde{\ell}_{1}(y), \tilde{\ell}_{2}(y)$ is multinomial (see the Appendix) and an application of the central limit theorem yields the following result. For large $\ell(0)$ the random vector $\left(\tilde{\xi}_{2}(y), \tilde{\xi}_{3}(y)\right)^{\prime}$ is approximately normally distributed with mean $\left(\xi_{2}(y), \xi_{3}(y)\right)^{\prime}$ and covariance matrix

$$
V(y)=\left(\begin{array}{cc}
\ell_{2}(y)^{-1}+\ell_{1}(y)^{-1} & \ell_{1}(y)^{-1} \\
\ell_{1}(y)^{-1} & \ell_{3}(y)^{-1}+\ell_{1}(y)^{-1}
\end{array}\right)
$$

A proof is given in the Appendix.

The usefulness of this result is that it is now possible to consider the vector regression

$$
\tilde{\xi}(y)=\xi(y)+\varepsilon(y)
$$

for a set of ages $y_{1}, \ldots, y_{n}$ to estimate parameterized $\xi(y)=\xi(y ; \beta)$. However, the error vectors $\varepsilon\left(y_{i}\right)$ are generally not independent and we follow the two-stage procedure given in Section 3 of Liang and Zeger [18]. The first step consists of using weighted least squares with a 'working' error covariance matrix to obtain a consistent estimator of the regression parameters. Standard errors of the estimates are obtained in the second stage and it is shown how this can be done in the Appendix.

To implement the first stage of the Liang-Zeger procedure, we use the working assumption that the error random vectors $\varepsilon\left(y_{i}\right), j=1, \ldots, n$ are independent with covariance matrices $V(y)$ of (7). For the Australian data discussed in Sections 3 and 4 it turns out that $\xi_{i}$ linear or quadratic in age $y$ provide an adequate description of the data. Suppose in fact that we adopt the models

$$
\begin{aligned}
& \xi_{2}=z^{\prime} \gamma=\sum_{r=1}^{k_{2}} z_{r} \gamma_{r} \\
& \xi_{3}=w^{\prime} \delta=\sum_{r=1}^{k_{3}} w_{r} \delta_{r}
\end{aligned}
$$

where $z$ and $w$ are known vectors depending on age and perhaps other variables. The vectors $\gamma$ and $\delta$ are to be estimated. Write

$$
\beta^{\prime}=\left(\gamma^{\prime}, \delta^{\prime}\right)
$$


If also

$$
Z(y)=\left(\begin{array}{cc}
z & 0 \\
0 & w
\end{array}\right)
$$

the regression to be estimated is

$$
\tilde{\xi}(y)=Z(y)^{\prime} \beta+\varepsilon(y)
$$

with $\varepsilon(y)$ bivariate normal with zero mean and covariance matrix $V(y)$. Under the working assumption of independence and with data available for a sequence of ages, the vector $\beta$ can be estimated by minimizing the weighted least squares loss function

$$
L(\beta)=\sum_{y}\left(\tilde{\xi}(y)-Z(y)^{\prime} \beta\right)^{\prime} V^{-1}(y)\left(\tilde{\xi}(y)-Z(y)^{\prime} \beta\right)
$$

The matrix of weights is a standard form

$$
V^{-1}(y)=\ell(0)\left(\begin{array}{cc}
p_{2}\left(1-p_{2}\right) & -p_{2} p_{3} \\
-p_{2} p_{3} & p_{3}\left(1-p_{3}\right)
\end{array}\right)
$$

and minimization presents no difficulties. For calculations, the $p_{i}$ are replaced by realizations of $\tilde{\ell}_{i}(y) / \ell(0)$.

With $\xi_{2}, \xi_{3}$ suitably parameterized the weighted least squares estimator $\hat{\beta}$ of $\beta$ is

$$
\hat{\beta}=\left(\sum_{y} Z(y) V^{-1}(y) Z(y)^{\prime}\right)^{-1}\left(\sum_{y} Z(y) V^{-1}(y) \tilde{\xi}(y)\right)
$$

It is (asymptotically) normally distributed with mean $\beta$ and covariance matrix that will be discussed in the Appendix. For each $y$ the estimates of the $\log$ (odds) are

$$
\hat{\xi}_{2}=z^{\prime} \hat{\gamma}, \quad \hat{\xi}_{3}=w^{\prime} \hat{\gamma}
$$

Using the definition of the $e_{i}(y)$ we can write down their respective estimators $\hat{e}_{i}(y)$ in terms of the $\hat{\gamma}, \hat{\delta}$ and the known vectors $z, w$. The latter have entries that typically include powers of $y$, such as $z^{\prime}=\left(1, y, y^{2}, I\right)$ for a quadratic in age and dummy variable $I$ that could be due to sex or a social indicator. The only constraints on the number of elements in $z$ and $w$ are the practical ones of data availability. The matrix $Z(y)$ then specifies the design of the regression in the usual sense.

If the initial frequencies $\ell_{1}(0)$ and $\ell_{2}(0)$ of the alive states are known then it can be shown that the approximating large sample normal distribution of $\tilde{\xi}(y)-\xi(y)$ has covariance matrix $V(y)+C(y)$ where $C(y)$ involves probabilities of transitions between states. These transition probabilities cannot be directly estimated from cross-sectional data. Therefore also in this case we continue with the working assumption adopted above and use weight matrix $V(y)^{-1}$ in the loss function (9).

The estimator of $p_{i}(y)$, obtained by substituting $\hat{\xi}_{2}(y), \hat{\xi}_{3}(y)$ into (5), will be written $p_{i}(y ; \hat{\beta})$. Returning to the notation of Section 2 with $p_{i}(x+y)$ the conditional probability of state $i$ at age $x+y$, given being alive at $x$, the estimated health expectancy of state $i$ 
at age $x$ is

$$
\hat{e}_{i}(x)=\sum_{y=0}^{\infty} p_{i}(x+y ; \hat{\beta})
$$

This is the formula used to obtain the numerical values of Section 4. Standard errors were calculated using (A3) of the Appendix.

\section{APPENDIX}

For large initial population counts, as in the data of Section 3, the following approximation in probability using the expansion for $\log (1+z)$ has useful consequences:

$$
\begin{aligned}
\tilde{\xi}_{i}-\xi_{i} & =\log \left\{\tilde{\ell}_{i} / \ell_{i}\right\}-\log \left\{\tilde{\ell}_{1} / \ell_{1}\right\} \\
& =\log \left[1+\left\{\tilde{\ell}_{i}-\ell_{i}\right\} / \ell_{i}\right]-\log \left[1+\left\{\tilde{\ell}_{1}-\ell_{1}\right\} / \ell_{1}(y)\right] \\
& \approx \frac{\tilde{\ell}_{i}}{\ell_{i}}-\frac{\tilde{\ell}_{1}}{\ell_{1}} \quad \text { as } \ell(0) \rightarrow \infty
\end{aligned}
$$

Hence assumptions made about the distribution of the counting random variables determine the large sample distribution of the $\tilde{\xi}_{i}(y)$. We give a justification of the key results in Section 5 .

It will be assumed that the usual life table assumption of homeogeneity holds, that is individuals in the same cohort with the same initial state stochastically evolve independently and identically as far as transitions between health states are concerned. The following is a special case of a more general result discussed by the authors elsewhere.

Result $A 1$. As $\ell(0) \rightarrow \infty$, the random vector $\tilde{\xi}(y)^{\prime}=\left(\tilde{\xi}_{2}(y), \tilde{\xi}_{3}(y)\right)^{\prime}$ is asymptotically normally distributed with mean $\left(\xi_{2}(y), \xi_{3}(y)\right)^{\prime}$ and covariance matrix (7).

Proof. The assumption of homogeneity implies that for each fixed $y$ the frequencies $\tilde{\ell}_{i}(y)=\tilde{\ell}_{i}$, $\sum_{i=1}^{3} \tilde{\ell}_{i}=\ell(0)$, are multinomially distributed with joint moment generating function

$$
E \exp \left\{\sum_{i=1}^{3} s_{i} \tilde{\ell}_{i}\right\}=\left\{\sum_{i=1}^{3} p_{i} \mathrm{e}^{S_{i}}\right\}^{\ell(0)}
$$

From (A1), as $\ell(0) \rightarrow \infty$

$$
\begin{gathered}
E \exp \left\{\sum_{i=2}^{3} s_{i} \ell(0)^{1 / 2}\left[\tilde{\xi}_{i}(y)-\xi_{i}(y)\right]\right\} \approx E \exp \left\{\sum_{i=2}^{3} s_{i} \ell(0)^{1 / 2}\left[\left(\tilde{\ell}_{i} / \ell_{i}\right)-\left(\tilde{\ell}_{1} / \ell_{1}\right)\right]\right\} \\
=\left\{p_{1} \exp \left[-\ell(0)^{-1 / 2} p_{1}^{-1}\left(\sum_{i=2}^{3} s_{i}\right)\right]+\sum_{i=2}^{3} p_{i} \exp \left[\ell(0)^{-1 / 2} p_{i}^{-1} s_{i}\right]\right\}^{\ell(0)}
\end{gathered}
$$


Expanding the exponentials and proceeding to the limit yields the moment generating function of the asserted bivariate normal distribution:

$$
\lim _{\ell(0) \rightarrow \infty} E \exp \left\{\sum_{i=2}^{3} s_{i} \ell(0)^{1 / 2}\left[\tilde{\xi}_{i}(y)-\xi_{i}(y)\right]\right\}=\exp \left\{\frac{1}{2} s^{\prime} \ell(0) V(y) s\right\}
$$

where $s^{\prime}=\left(s_{2}, s_{3}\right)$ and $V(y)$ is given by (7).

Moment generating functions can also be used to establish the asymptotic joint normality of $\ell(0)^{1 / 2}\{\tilde{\xi}(u)-\xi(u)\}$ and $\ell(0)^{1 / 2}\{\tilde{\xi}(y)-\xi(y)\}$. In general they are not independent and also the asymptotic covariance $\sigma_{i j}(u, y)=\lim _{\ell(0) \rightarrow \infty}\left\{\ell(0) \operatorname{cov}\left(\tilde{\xi}_{i}(u), \tilde{\xi}_{j}(y)\right)\right\}$ typically depend on transition probabilities which cannot be directly estimated from cross-sectional data. We argue as follows.

From (11) the variance of $\hat{e}_{i}(x)$ is

$$
\operatorname{var}\left\{\hat{e}_{i}(x)\right\}=\sum_{y=0}^{\infty} \operatorname{var}\left\{p_{i}(x+y ; \hat{\beta})\right\}+2 \sum_{u<y} \sum \operatorname{cov}\left\{p_{i}(x+u ; \hat{\beta}), p_{i}(x+y ; \hat{\beta})\right\}
$$

The delta method can be used to obtain

$$
\begin{aligned}
& \operatorname{var}\left\{p_{i}(x+y ; \hat{\beta})\right\}=\left(\frac{\partial}{\partial \beta} p_{i}(x+y ; \beta)\right)^{\prime}(\operatorname{var} \hat{\beta})\left(\frac{\partial}{\partial \beta} p_{i}(x+y ; \beta)\right) \\
& \operatorname{cov}\left\{p_{i}(x+u ; \hat{\beta}), p_{i}(x+y ; \hat{\beta})\right\}=\left(\frac{\partial}{\partial \beta} p_{i}(x+u ; \beta)\right)(\operatorname{var} \hat{\beta})\left(\frac{\partial}{\partial \beta} p_{i}(x+y ; \beta)\right)
\end{aligned}
$$

There remains the problem of estimating the covariance matrix of $\hat{\beta}$ and we utilize Theorem 2 of Liang and Zeger [18]. At issue is dealing with dependence within cohorts as age increases and direct estimation of this dependence is not possible from cross-sectional data. Since cohorts are assumed independent, a reformulation in these terms is necessary. There are only five cohorts but the number in each is large and the Liang-Zeger asymptotics must be modified accordingly. The standard errors of Table III were obtained in this way. Details of the argument are omitted.

The following result gives the asymptotic joint distribution of the estimators of health expectancy $\hat{e}_{1}(x)$ and $\hat{e}_{2}(x)$.

Result 2. The estimators $\hat{e}_{1}(x)$ and $\hat{e}_{2}(x)$ of health expectancy for states 1 and 2 given by

$$
\hat{e}_{i}(x)=\sum_{y=0}^{\infty} p_{i}(x+y ; \hat{\beta})
$$

are, as $\ell(0) \rightarrow \infty$, asymptotically bivariate normally distributed with means $e_{1}(x), e_{2}(x)$ and covariance matrix given by equations (A3) and (A4).

Proof. For $i=1,2$ the delta method uses a first-order Taylor expansion for $p_{i}(x+y ; \hat{\beta})$

$$
p_{i}(x+y ; \hat{\beta})=p_{i}(x+y ; \beta)+(\hat{\beta}-\beta)^{\prime}\left(\frac{\partial p_{i}(x+y ; \beta)}{\partial \beta}\right)
$$

Since $\hat{\beta}-\beta$ is asymptotically normally distributed with mean the zero vector and covariance matrix $\operatorname{var} \hat{\beta}$ then $p_{1}(x+y ; \hat{\beta}), p_{2}(x+y ; \hat{\beta})$ are asymptotically jointly normally distributed 
with means $p_{1}(x+y ; \beta), p_{2}(x+y ; \beta)$ and second moments (A4). The asserted result follows immediately.

We point out that the derivatives of the probabilities with respect to $\beta$ can be easily found.

For example, if $z^{\prime} \gamma=\gamma_{0}+x \gamma_{1}$, then

$$
\frac{\partial}{\partial \gamma_{1}} p_{1}(x ; \beta)=-x p_{1}^{2}(x ; \beta) \exp \left(z^{\prime} \gamma\right)=-x p_{1}(x ; \beta) p_{2}(x ; \beta)
$$

\section{ACKNOWLEDGEMENTS}

One author (C. R. Heathcote) wishes to acknowledge assistance and hospitality provided by the Department of Epidemiology and Public Health, University of Leicester. An earlier version of the paper was read at the April 1999 London meeting REVES 11 (Réseau Espérance de Vie en Santé). Also, we are indebted to Dr C. Mathers for kindly making available unpublished details of the results of the 1993 survey and to the referees for constructive comments.

\section{REFERENCES}

1. Katz S, Branch LG, Bramson MH, Papsidero JA, Beck JC, Greer DS. Active life expectancy. New England Journal of Medicine 1983; 309:1218-1224.

2. Bebbington AC. The expectation of life without disability in England and Wales. Social Science in Medicine 1988; 27:321-326.

3. Robine J-M, Ritchie K. Healthy life expectancy: evaluation of global indicator of change in population health. British Medical Journal 1991; 302:457-460.

4. Mathers C, McCallum J, Robine J-M (eds). Advances in Health Expectancies. Australian Institue of Health and Welfare: Canberra, 1994.

5. Mathers C. Trends in health expectancies in Australia 1981-1993. Journal of the Australian Population Association 1996; 13:1-15.

6. Mathers C. Health Expectancies in Australia 1981 and 1988. Australian Institue of Health: Canberra, 1991.

7. Sullivan DF. A single index of mortality and morbidity. Health Services and Mental Health Administration Health Reports $1971 ; \mathbf{8 6}: 347-354$.

8. Deaton A. Panel data from time series of cross-sections. Journal of Econometrics 1985;30:109-126.

9. MacRae EC. Estimation of time-varying Markov processes with aggregate data. Econometrica 1977; 45:183-198.

10. Gargiullo PM, Rothenberg RB, Wilson HG. Confidence intervals, hypothesis tests, and sample sizes for the prevented fraction in cross-sectional studies. Statistics in Medicine 1995; 14:51-72.

11. Martuzzi M, Elliott P. Estimating the incidence rate ratio in cross sectional studies using a simple alternative to logistic regression. Annals of Epidemiology 1998; 8:52-55.

12. Thompson ML. Myers JE, Kriebel D. Prevalence odds ratio or prevalence ratio in the analysis of cross sectional data - what is to be done. Occupational and Environmental Medicine 1998; 55:272-277.

13. Skov T, Deddens J, Petersen MR, Endahl L. Prevalence proportion ratios - estimation and hypothesis testing. International Journal of Epidemiology 1998; 27:91-95.

14. Zocchetti C, Consonni D, Bertazzi PA. Relationship between prevalence rate ratios and odds ratios in crosssectional studies. International Journal of Epidemiology 1997; 26:220-223.

15. Land KC, Guralnik JM, Blazer DG. Estimating increment - decrement life tables with multiple covariates from panel data: the case of active life expectancy. Demography 1994; 31:297-319.

16. Chiang CC. Introduction to Stochastic Processes in Biostatistics. Wiley: New York, 1968.

17. Olshansky SJ, Rudberg MA, Carnes BA, Cassel CK, Brody JA. Trading off longer life for worsening health. Journal of Aging and Health 1991; 3:194-216.

18. Liang KY, Zeger SL. Longitudinal data analysis using generalised linear models. Biometrika 1986; 73:13-22. 